\title{
Clinicopathologic Features and Long-Term Outcomes of Elderly Breast Cancer Patients: Experiences at a Single Institution in Korea
}

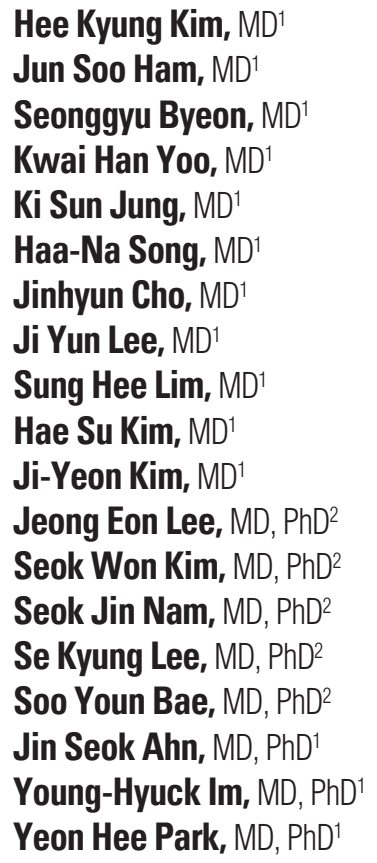

${ }^{1}$ Division of Hematology-Oncology, Department of Internal Medicine, ${ }^{2}$ Department of Surgery, Samsung Medical Center, Sungkyunkwan University School of Medicine, Seoul, Korea

\begin{abstract}
Purpose
The purpose of this study was to assess the tumor characteristics and long-term clinical outcomes of adjuvant treatments after surgery with a curative aim for patients with breast cancer who are 65 years and older.
\end{abstract}

\section{Materials and Methods}

Patients with breast cancer who underwent curative surgery from 2000 to 2009 were analyzed $(n=4,388)$. Tumor characteristics and survival outcome were compared by dividing the patients into two age groups ( $<65$ and $\geq 65$ years old). The Kaplan-Meier method was used for comparison of survival rates by log-rank test, and a Cox regression model was used to examine the effect of variables.

\section{Results}

Among 4,388 patients with invasive breast cancer, 317 patients (7.2\%) were 65 years or older and the median age of all patients was 47 years (range, 18 to 91 years). Tumor characteristics were similar between the two age groups, but the older patients were treated less often with adjuvant treatments. During a median follow-up period of 122 months, recurrence-free survival (RFS) was equivalent for patients 65 years and older compared to younger patients, but significantly worse in overall survival (OS) and breast cancer-specific survival (BCSS) (5-year OS, 94.3\% vs. 90.5\%; p < 0.001 and 5-year BCSS, 94.7\% vs. 91.8\%; $\mathrm{p}=0.031$ ). In the multivariate model, age $\geq 65$ years old was identified as an independent risk factor for OS and RFS.

\section{Conclusion}

Elderly breast cancer appeared to have worse outcomes with very low prevalence in Korea, despite similar tumor characteristics. More active adjuvant therapies would have a role for aggressive subtypes for fit, elderly patients.
Correspondence: Yeon Hee Park, MD, PhD

Division of Hematology-Oncology,

Department of Medicine,

Samsung Medical Center,

Sungkyunkwan University School of Medicine,

81 Irwon-ro, Gangnam-gu, Seoul 06351, Korea

Tel: 82-2-3410-1780

Fax: 82-2-3410-1754

E-mail: yhparkhmo@skku.edu

Received November 2, 2015

Accepted March 3, 2016

Published Online March 11, 2016

\author{
Key words \\ Breast neoplasms, Aged, Population characteristics, \\ Treatment outcome
}




\section{Introduction}

The elderly population is increasing worldwide. The population of 65 years or older among all cancer patients is anticipated to be $20.1 \%$ (70.2 million) of the total by 2030 in the United States [1], and 24.3\% (12.6 million) in Korea [2]. Breast cancer is the most common cancer among women [3], and the number of older women with breast cancer is increasing. However, demographic features of patients with breast cancer differ between Asian and Western countries. Asian women, including Korean women, have a lower incidence rate and younger peak age of breast cancer compared with the Caucasian population $[4,5]$. The incidence of breast cancer in women peaks in their forties in Asia, but the peak age in the United States is in their sixties [6]. Fig. 1 shows that the incidence rates increase with age for most cancers, but that the peak incidence age of breast cancer was between 45 and 59 years in the Korean population [7].

As a consequence of the high incidence of young age breast cancer in Asia, a number of studies investigated young breast cancer including early stage breast cancer. However, older patients with breast cancer presented less with active treatments such as clinical trials [8]. Although most elderly patients with breast cancer present at an early stage [9], few studies on the clinical outcome and tumor biology of elderly patients with early stage breast cancer have been reported.

Comorbidity and functional status of elderly patients could result in poorer survival, but there are considerable factors that render positive outcomes in older patients. First, breast cancer in postmenopausal women shows a slowgrowing and indolent nature [10]. Recently, the improvement of general health has increased the life expectancy to around 80 years in many countries, so that survival outcome could be comparable to that of younger patients in early stage breast cancer. However, the significance of early breast cancer on the survival of elderly patients is still unknown. Elderly breast cancer patients are defined as breast cancer patients aged 65 and over. We have chosen 65 years or older, however there is no consensus to define elderly breast cancer, because of the extremely low incidence for women 70 years or older in our patients' cohort $(3.6 \%, 159 / 4,388)$ and some geriatric recommendations $[11,12]$.

Optimal treatment for older breast cancer patients is also not well established [13]. The treatment approach for elderly patients requires many considerations, including not only chronological age but also comorbidities, social and economic circumstances, and life expectancy. However, the majority of recommendations are based on retrospective analyses and the extrapolation of study results from younger patients. This may result in under treatment and poor survival outcomes in the older populations [14].

In this study, we have explored the biologic tumor characteristics and clinical outcomes of elderly women (65 years and older than 65 years of age) with early stage breast cancer. In addition, we compared the results with those of patients younger than 65 years old, with analysis of long-term outcome and survival rate. Then, our results were compared with those of Western countries.

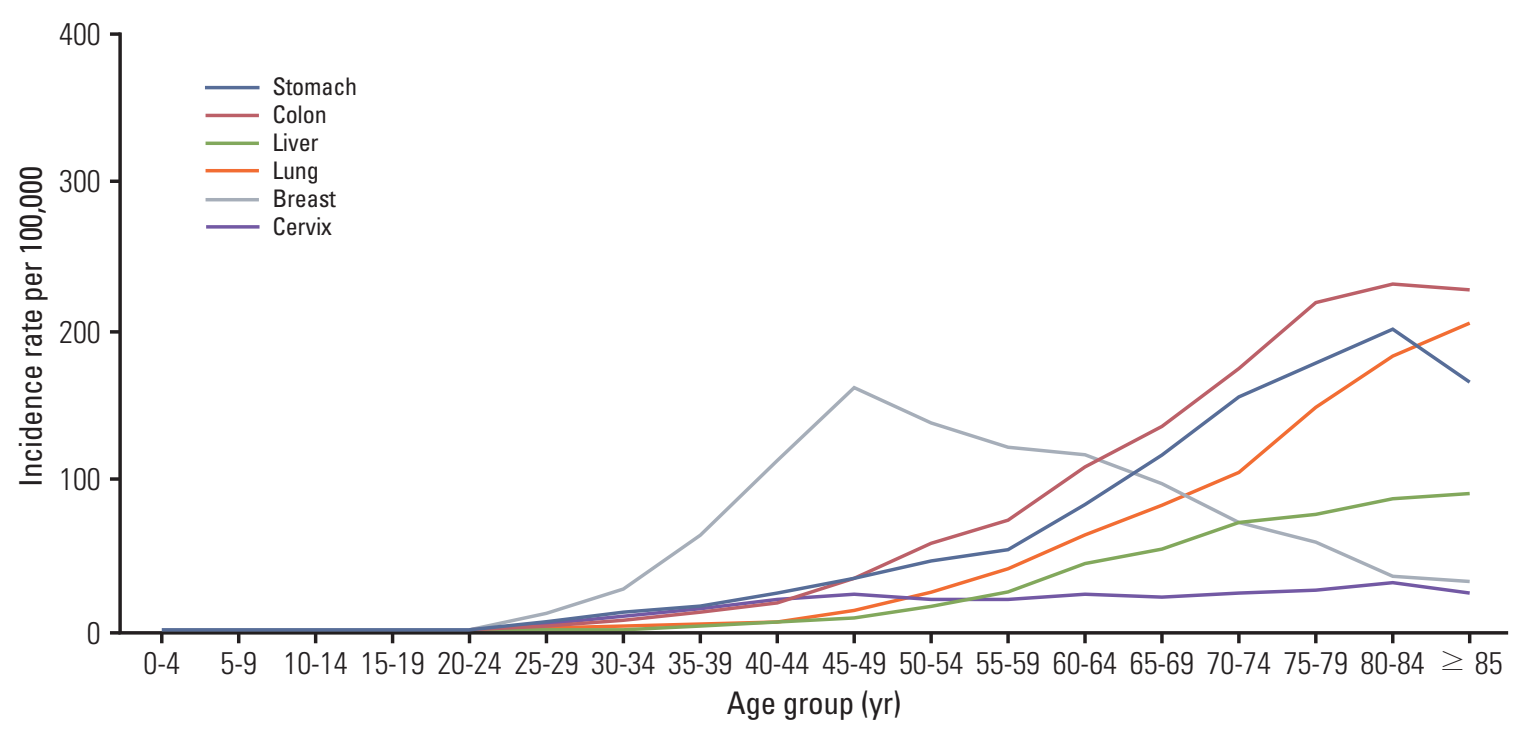

Fig. 1. Age-specific cancer incidence of women in Korea. 


\section{Materials and Methods}

\section{Patients}

In this retrospective cohort study, we collected data from the electronic medical records of patients diagnosed with invasive breast cancer who underwent curative surgery at Samsung Medical Center between 2000 and 2009. Data including TNM staging, the results of estrogen/progesterone/HER2 expression, nuclear grade, histologic grade, Ki-67 expression, lymphatic and vascular invasion, date of surgery, and all adjuvant therapies (including systemic chemotherapy, endocrine therapy, and radiotherapy) were collected for this study. The patients were divided into two age groups, $<65$ years old and $\geq 65$ years old. Intrinsic subtypes were classified according to four groups based on immunohistochemistry as follows: hormone receptor (HR)+/ HER2-, HR+/HER2+, HR-/HER2+, and triple negative breast cancer. For detection of local or distant recurrence, clinical follow-up was performed every 3-6 months for the first 5 years after primary therapy and annually thereafter. Clinical follow-up included history-taking; physical examinations; laboratory tests, including carcinoembryonic antigen, cancer antigen 15-3, complete blood counts, and liver function tests; chest radiography; mammography; breast and abdominopelvic ultrasonography; and bone scans. In addition, a computed tomography (CT) scan, magnetic resonance imaging, or a fluorine-18 fluorodeoxyglucose positron emission tomography/CT scan was performed if necessary. This study was approved by the Institutional Review Board of Samsung Medical Center, Seoul, Korea.

\section{Statistical analysis}

Differences in characteristics including distribution of intrinsic subtypes between two age groups ( $<65$ years vs. $\geq 65$ years) were examined using Fisher exact test. Overall survival (OS) was measured from the date of curative surgery to the date of death or date the patient was last seen. Recurrence-free survival (RFS) was measured from the date of curative surgery to the date of breast cancer recurrence, regardless of whether recurrence was locoregional, contralateral breast and/ or distant metastasis. Distant RFS (DRFS) was defined as the time from the date of curative surgery to the date of documented distant metastasis. Breast cancer-specific survival (BCSS) was determined from only breast cancer-specific death. The Kaplan-Meier method was used for estimation of OS, RFS, DRFS, and BCSS. Differences in survival were analyzed using the log-rank test and a p-value less than 0.05 was considered significant. A multivariable Cox proportional hazard regression model was used to assess the impact of the prognostic variable on OS and RFS. Data were analyzed using the IBM SPSS ver. 22.0 software (IBM Co., Armonk, NY).

\section{Results}

A total of 4,409 female patients who underwent curative surgery were diagnosed with invasive breast cancer between 2000 and 2009. Among them, 21 patients with metastatic breast cancer (stage IV) were identified and excluded from analysis. Only 317 patients $(7.2 \%)$ were 65 years or older, while the rest of the patients were younger than 65 years old. The median ages of the younger and older groups were 46 years (range, 18 to 65 years) and 70 years (range, 65 to 91 years), respectively. There was no significant difference between the two groups according to TNM staging, nuclear grade, histologic grade, or Ki-67 expression. Lymphovascular invasion was more frequent in the younger age group (33.7\% vs $24.9 \%, \mathrm{p}=0.015)$. Older patients received adjuvant treatments, including local and systemic therapies for breast cancer, less often (Table 1). The majority of the younger age group were treated with adjuvant chemotherapy and radiotherapy $(80.4 \%$ and $68.8 \%$, respectively), whereas older patients were treated less often $(46.7 \%$ and $48.3 \%$, respectively). In the case of the adjuvant endocrine therapy, more than half of the older patients $(51.7 \%)$ were prescribed adjuvant endocrine therapy. Patients with HER2+ had received adjuvant trastuzumab since 2008 , because insurance did not reimburse the cost until that time. The majority of younger patients with HER2+ $(73.2 \%)$ were treated with adjuvant trastuzumab, while only $27.3 \%$ of older patients received adjuvant trastuzumab treatment.

The 5-year RFS rate and OS rate in all patients $(n=4,388)$ were $87.4 \%$ and $94.1 \%$, respectively, with a median followup period of 122 months. Fig. 2 shows the Kaplan-Meier curves by RFS and DRFS, OS, and BCSS, according to age group. There were no significant differences in RFS and DRFS according to age group; however, the OS and BCSS differed between age groups. The results of univariate analysis for RFS and OS with a 5-year survival rate according to each variable are shown in Supplementary Tables 1 and 2.

In multivariate Cox-regression analysis, old age ( $\geq 65$ years), TNM staging, and lymphovascular invasion were identified as independent risk factors for RFS, DRFS, and OS. However, in terms of BCSS, old age was not an independent prognostic factor (hazard ratio, 0.079; 95\% confidence interval, 0.94 to $3.21 ; \mathrm{p}=0.079$ ). Among the adjuvant therapies, adjuvant endocrine therapy was a positive prognostic factor in all survival analyses (Table 2) 
Table 1. Patients' characteristics

\begin{tabular}{|c|c|c|c|}
\hline Variable & Age $<65$ yr $(n=4,071)$ & Age $\geq 65$ yr $(n=317)$ & p-value \\
\hline Age (yr) & $46(18-65)$ & $70(65-91)$ & \\
\hline \multicolumn{4}{|l|}{ Stage at diagnosis } \\
\hline I & $1,630(40.0)$ & $120(37.9)$ & 0.580 \\
\hline II & $1,765(43.4)$ & $147(46.4)$ & \\
\hline III & $676(16.6)$ & $50(15.8)$ & \\
\hline Proportion of IDC & $3,402(83.6)$ & $268(84.5)$ & 0.651 \\
\hline \multicolumn{4}{|l|}{ Nuclear grade $(n=4,216)$} \\
\hline I & $519(13.3)$ & $50(16.3)$ & 0.323 \\
\hline II & $1,703(43.6)$ & $131(42.7)$ & \\
\hline III & $1,687(43.2)$ & $126(41.0)$ & \\
\hline \multicolumn{4}{|l|}{ Histologic grade $(n=3,982)$} \\
\hline Low & $695(18.8)$ & $59(20.6)$ & 0.765 \\
\hline Intermediate & $1,586(42.9)$ & $120(41.8)$ & \\
\hline High & $1,414(38.3)$ & $108(37.6)$ & \\
\hline \multicolumn{4}{|l|}{ Ki-67 (\%) (n=2,406) } \\
\hline$<50$ & $1,566(70.8)$ & $131(67.9)$ & 0.399 \\
\hline$\geq 50$ & $647(29.2)$ & $62(32.1)$ & \\
\hline Lymphovascular invasion $(n=1,797)$ & $541(33.7)$ & $48(24.9)$ & 0.015 \\
\hline \multicolumn{4}{|l|}{ Adjuvant chemotherapy } \\
\hline Yes & $3,273(80.4)$ & $148(46.7)$ & $<0.001$ \\
\hline No & 797 (19.6) & $169(53.3)$ & \\
\hline \multicolumn{4}{|l|}{ Adjuvant radiotherapy } \\
\hline Yes & $2,800(68.8)$ & $153(48.3)$ & $<0.001$ \\
\hline No & $1,270(31.2)$ & $164(51.7)$ & \\
\hline \multicolumn{4}{|l|}{ Adjuvant endocrine therapy } \\
\hline Yes & $2,661(65.4)$ & $182(57.4)$ & 0.004 \\
\hline No & $1,409(34.6)$ & $135(42.6)$ & \\
\hline \multicolumn{4}{|l|}{ Adjuvant trastuzumab (n=224) } \\
\hline Yes & $90(73.2)$ & $3(27.3)$ & 0.003 \\
\hline No & $33(26.8)$ & $8(72.7)$ & \\
\hline
\end{tabular}

Values are presented as median (range) or number (\%). IDC, invasive ductal carcinoma.

The distribution of intrinsic subtypes was almost identical between the two age groups $(\mathrm{p}=0.541)$, and the most common subtype was HR(+)/HER2(-), 59.4\% and 57.6\%, respectively (Fig. 3). In univariate analysis, intrinsic subtypes among younger patients (age $<65$ years) were identified as risk factors in terms of RFS and OS (Supplementary Tables 1 and 2). Intrinsic subtypes were excluded as a variable in the multivariate Cox-regression analysis because it could be confounded with affected adjuvant endocrine therapy.

\section{Discussion}

Almost $50 \%$ of female patients with breast cancer are age
65 years or older in the United States with a median age of diagnosis at 61 years $[15,16]$. In the current study, this was in stark contrast with western countries where only $7.2 \%$ of patients in our cohort were 65 years or over, with the median age of all patients of 47 years (range, 18 to 91 years). Some differences between Asian and Western women, including breast density and HR, were identified [17], and further investigation of the clinical data regarding these differences is required.

Even though breast cancer in older women was known for its indolent nature $[10,16]$, most tumor characteristics were similar between women in the two age groups in our study, except for lymphovascular invasion. The incidence of lymphovascular invasion was lower in older patients (24.9\% vs. $33.7 \%, \mathrm{p}=0.015)$; however, the evidence was too weak to support that breast cancer in older patients is less aggressive 

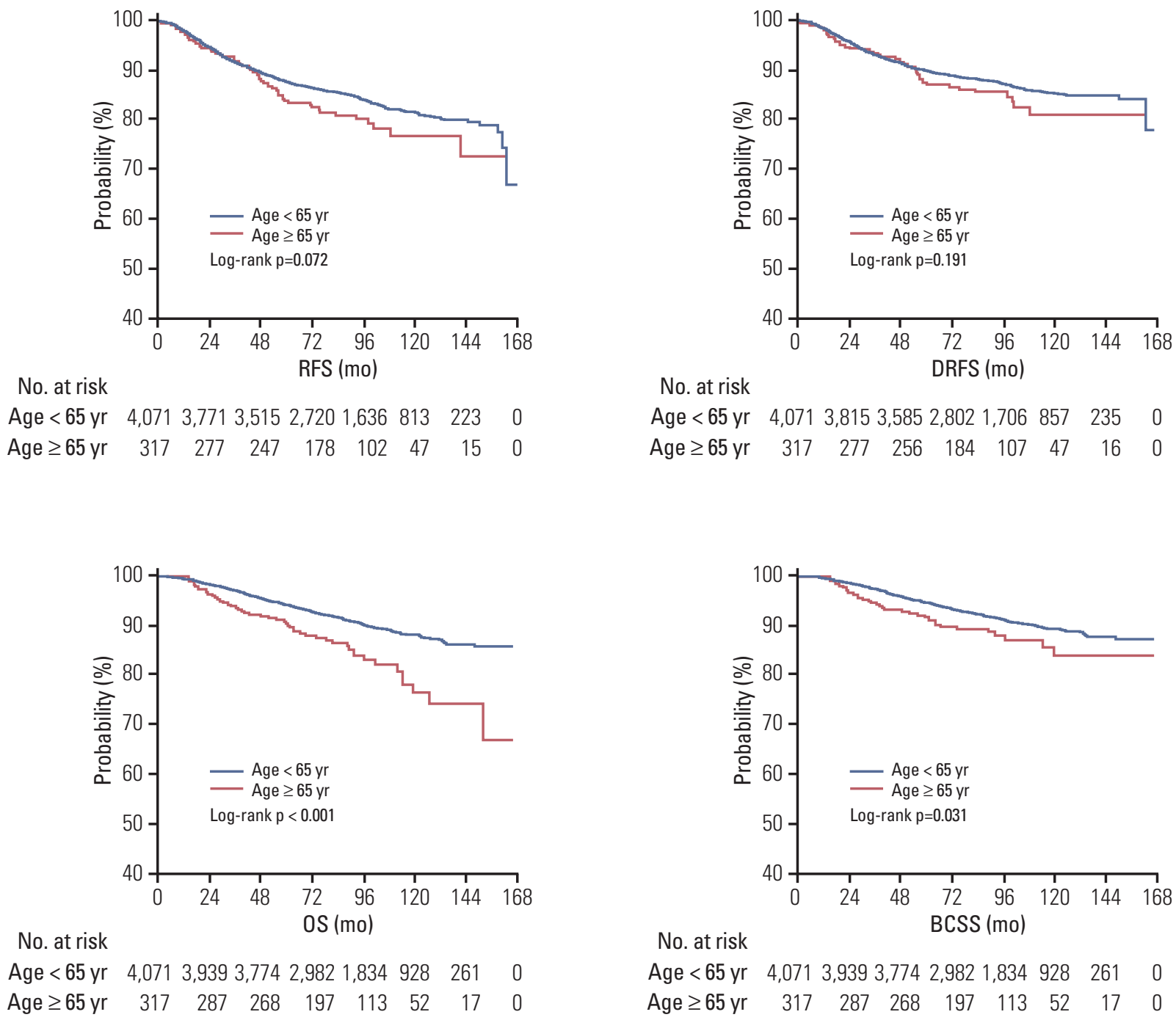

Fig. 2. Recurrence-free survival (RFS), distant recurrence-free survival (DRFS), overall survival (OS), and breast cancer-specific survival (BCSS) according to age groups.

than in younger patients; this is because the other tumor characteristics, including nuclear grade, histologic grade, and expression of Ki-67, were not significantly different between the two age groups in this study.

This study showed that older patients were less likely to receive adjuvant treatments after curative surgery. The discrepancy between the two age groups was largest for adjuvant chemotherapy ( $48.3 \%$ vs. $80.4 \%, p<0.001)$, because the comorbidity or disability in elderly patients with breast cancer poses a challenge for aggressive treatment. This finding is similar to those of studies in western countries $[9,14,18]$. However, endocrine therapy tended to be administered more often to older patients than other adjuvant therapies ( $57.4 \%$ vs. $65.4 \%$, $\mathrm{p}=0.004)$, and adjuvant endocrine therapy was an independent, favorable prognostic factor in all survival analyses. Because the percentages of HRs were not significantly different between the two age groups, our findings suggest that all patients with breast cancer who have undergone curative surgery benefit from adjuvant endocrine therapy, regardless of age.

Several randomized trials confirmed the effectiveness of adjuvant chemotherapy and radiotherapy in improving survival for older women with early-stage breast cancer [19-21]. Under treatment of elderly breast cancer was demonstrated in several studies and typically resulted in worse clinical outcome $[14,18]$. Older patients in our cohort had less adjuvant therapies, and breast cancer-specific survival was lower than for younger patients $(94.7 \%$ vs. $91.8 \%$ BCSS at 5 years, $89.5 \%$ 
Table 2. Multivariate Cox-regression model

\begin{tabular}{|c|c|c|c|c|c|c|c|c|}
\hline \multirow{2}{*}{ Variable } & \multicolumn{2}{|c|}{ RFS } & \multicolumn{2}{|c|}{ DRFS } & \multicolumn{2}{|c|}{ OS } & \multicolumn{2}{|c|}{ BCSS } \\
\hline & $\mathrm{HR}(95 \% \mathrm{CI})$ & p-value & $\mathrm{HR}(95 \% \mathrm{CI})$ & p-value & $\mathrm{HR}(95 \% \mathrm{CI})$ & p-value & HR $(95 \% \mathrm{CI})$ & p-value \\
\hline Age $\geq 65 \mathrm{yr}$ & $\begin{array}{c}1.68 \\
(1.11-2.55)\end{array}$ & 0.015 & $\begin{array}{c}2.48 \\
(1.09-2.86)\end{array}$ & 0.022 & $\begin{array}{c}1.91 \\
(1.09-3.36)\end{array}$ & 0.024 & $\begin{array}{c}1.73 \\
(0.94-3.21)\end{array}$ & 0.077 \\
\hline TNM staging & $\begin{array}{c}4.31 \\
(1.66-2.72)\end{array}$ & $<0.001$ & $\begin{array}{c}6.59 \\
(1.95-3.54)\end{array}$ & $<0.001$ & $\begin{array}{c}3.17 \\
(2.19-4.56)\end{array}$ & $<0.001$ & $\begin{array}{c}3.83 \\
(2.54-5.77)\end{array}$ & $<0.001$ \\
\hline Lymphovascular invasion & $\begin{array}{c}1.98 \\
(1.41-2.77)\end{array}$ & $<0.001$ & $\begin{array}{c}2.48 \\
(1.66-3.68)\end{array}$ & $<0.001$ & $\begin{array}{c}1.86 \\
(1.14-3.03)\end{array}$ & 0.013 & $\begin{array}{c}2.15 \\
(1.27-3.63)\end{array}$ & 0.005 \\
\hline Adjuvant chemotherapy & $\begin{array}{c}0.62 \\
(0.39-0.92)\end{array}$ & 0.018 & $\begin{array}{c}0.75 \\
(0.43-1.25)\end{array}$ & 0.299 & $\begin{array}{c}0.52 \\
(0.28-0.96)\end{array}$ & 0.035 & $\begin{array}{c}0.55 \\
(0.28-1.07)\end{array}$ & 0.082 \\
\hline Adjuvant radiotherapy & $\begin{array}{c}0.79 \\
(0.58-1.16)\end{array}$ & 0.260 & $\begin{array}{c}0.69 \\
(0.47-1.08)\end{array}$ & 0.098 & $\begin{array}{c}0.85 \\
(0.49-1.45)\end{array}$ & 0.555 & $\begin{array}{c}0.73 \\
(0.41-1.31)\end{array}$ & 0.296 \\
\hline Adjuvant endocrine therapy & $\begin{array}{c}0.47 \\
(0.34-0.65)\end{array}$ & $<0.001$ & $\begin{array}{c}0.42 \\
(0.29-0.60)\end{array}$ & $<0.001$ & $\begin{array}{c}0.19 \\
(0.12-0.31)\end{array}$ & $<0.001$ & $\begin{array}{c}0.19 \\
(0.12-0.31)\end{array}$ & $<0.001$ \\
\hline
\end{tabular}

RFS, recurrence-free survival; DRFS, distant recurrence-free survival; OS, overall survival; BCSS, breast cancer-specific survival; $\mathrm{HR}$, hazard ratio; $\mathrm{CI}$, confidence interval.

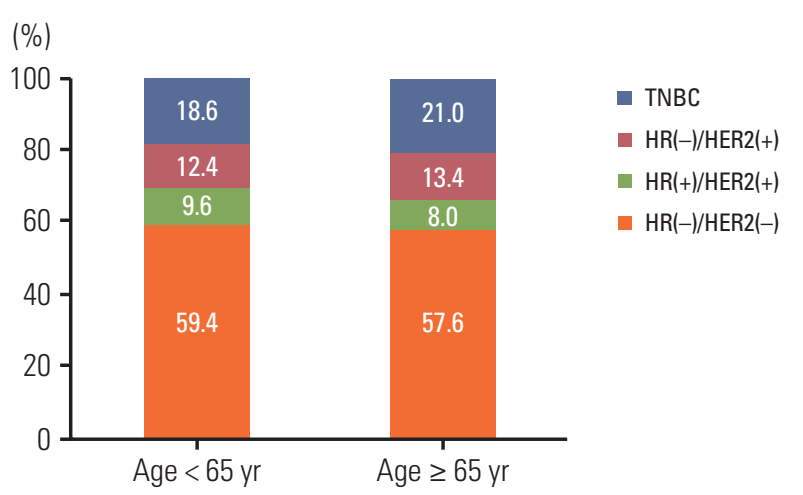

Fig. 3. Subtype distribution according to age groups. TNBC, triple negative breast cancer; HR, hormone receptor.

vs. $85.7 \%$ BCSS at 10 years); this implies that age-adjusted BCSS was worse for older patients. In addition, age 65 years or older was an independent risk factor for OS, but not for BCSS in multivariate analysis. These findings support that older patients should receive the standard treatment as is typically given to younger patients when possible. However, the lack of clinical information on comorbidity and performance status for comprehensive geriatric assessment has weakened our results.

In the current study, it would appear that worse long-term OS in patients aged 65 years or older is related to increasing comorbidity and disability according to age. Differences between OS and BCSS in older patients also suggest that patients aged 65 or older die of causes other than their breast cancer. Functional status is a crucial factor for adjuvant treatments in the elderly and several scales of geriatric assessment have been developed $[12,22,23]$. The clinician should use these tools in identification of vulnerable patients from healthy patients and to avoid suboptimal treatments for fit, elderly patients. According to increased longevity, a large number of older women are in good health, thus they may benefit just as much from aggressive treatment as younger patients. In addition, this study confirms that the median age is still younger than those of Western countries, even in the older group in our patients' cohort.

\section{Conclusion}

Only $7.5 \%$ of patients in our patients' cohort were elderly breast cancer patients aged 65 years or older, and the tumor characteristics were similar between the two age groups ( $<65$ and $\geq 65$ years old). However, most of the older patients were treated less often, even though they were in early stage of breast cancer. Further studies are needed to establish optimal treatment for elderly patients with breast cancer and to establish geriatric assessment for identification of high risk patients of treatment-related side effects. In addition, exploratory analysis for differentiation of biologic features between the two groups is warranted. 


\section{Electronic Supplementary Material}

Supplementary materials are available at Cancer Research and Treatment website (http://www.e-crt.org).

\section{Conflicts of Interest}

Conflict of interest relevant to this article was not reported.

\section{References}

1. Yancik R. Cancer burden in the aged: an epidemiologic and demographic overview. Cancer. 1997;80:1273-83.

2. Korea National Statistical Office. 2015 Statistical report on the aged [Internet]. Daejeon: Korea Statistical Information System (KOSIS); c2015 [cited 2015 Oct 1]. Available from: http:// kosis.kr/ups/ups_01List.jsp.

3. Ferlay J, Shin HR, Bray F, Forman D, Mathers C, Parkin DM. Estimates of worldwide burden of cancer in 2008: GLOBOCAN 2008. Int J Cancer. 2010;127:2893-917.

4. Han W, Kim SW, Park IA, Kang D, Kim SW, Youn YK, et al. Young age: an independent risk factor for disease-free survival in women with operable breast cancer. BMC Cancer. 2004;4:82.

5. Ko SS; Korean Breast Cancer Society. Chronological changing patterns of clinical characteristics of Korean breast cancer patients during 10 years (1996-2006) using nationwide breast cancer registration on-line program: biannual update. J Surg Oncol. 2008;98:318-23.

6. Leong SP, Shen ZZ, Liu TJ, Agarwal G, Tajima T, Paik NS, et al. Is breast cancer the same disease in Asian and Western countries? World J Surg. 2010;34:2308-24.

7. National Cancer Center. 2012 Report on the statistics for national cancer incidence [Internet]. Goyang: National Cancer Infromation Center; c2015 [cited 2015 Oct 1]. Available from: http://ncc.re.kr/ cancerStatsView.ncc?bbsnum=316\&searchKe $\mathrm{y}=$ total\&searchValue $=\&$ pageNum $=1$.

8. Goodwin JS, Hunt WC, Humble CG, Key CR, Samet JM. Cancer treatment protocols: who gets chosen? Arch Intern Med. 1988;148:2258-60.

9. Yancik R, Wesley MN, Ries LA, Havlik RJ, Edwards BK, Yates JW. Effect of age and comorbidity in postmenopausal breast cancer patients aged 55 years and older. JAMA. 2001;285: 885-92.

10. Clark GM. The biology of breast cancer in older women. J Gerontol. 1992;47 Spec No:19-23.

11. Karuturi M, VanderWalde N, Muss H. Approach and management of breast cancer in the elderly. Clin Geriatr Med. 2016;32:133-53.

12. Mislang AR, Biganzoli L. Adjuvant systemic therapy in older breast cancer women: can we optimize the level of care? Cancers (Basel). 2015;7:1191-214.

13. Biganzoli L, Wildiers H, Oakman C, Marotti L, Loibl S, Kun- kler I, et al. Management of elderly patients with breast cancer: updated recommendations of the International Society of Geriatric Oncology (SIOG) and European Society of Breast Cancer Specialists (EUSOMA). Lancet Oncol. 2012;13:e148-60.

14. Bouchardy C, Rapiti E, Fioretta G, Laissue P, Neyroud-Caspar I, Schafer $\mathrm{P}$, et al. Undertreatment strongly decreases prognosis of breast cancer in elderly women. J Clin Oncol. 2003;21: 3580-7.

15. Tew WP, Muss HB, Kimmick GG, Von Gruenigen VE, Lichtman SM. Breast and ovarian cancer in the older woman. J Clin Oncol. 2014;32:2553-61.

16. Diab SG, Elledge RM, Clark GM. Tumor characteristics and clinical outcome of elderly women with breast cancer. J Natl Cancer Inst. 2000;92:550-6.

17. Lawson JS, Field AS, Champion S, Tran D, Ishikura H, Trichopoulos D. Low oestrogen receptor alpha expression in normal breast tissue underlies low breast cancer incidence in Japan. Lancet. 1999;354:1787-8.

18. Bouchardy C, Rapiti E, Blagojevic S, Vlastos AT, Vlastos G. Older female cancer patients: importance, causes, and consequences of undertreatment. J Clin Oncol. 2007;25:1858-69.

19. Dore M, Cutuli B, Cellier P, Campion L, Le Blanc M. Hypofractionated irradiation in elderly patients with breast cancer after breast conserving surgery and mastectomy: analysis of 205 cases. Radiat Oncol. 2015;10:161.

20. Du XL, Zhang Y, Parikh RC, Lairson DR, Cai Y. Comparative effectiveness of chemotherapy regimens in prolonging survival for two large population-based cohorts of elderly adults with breast and colon cancer in 1992-2009. J Am Geriatr Soc. 2015;63:1570-82.

21. Muss HB, Berry DA, Cirrincione CT, Theodoulou M, Mauer AM, Kornblith AB, et al. Adjuvant chemotherapy in older women with early-stage breast cancer. N Engl J Med. 2009; 360:2055-65.

22. Klepin H, Mohile S, Hurria A. Geriatric assessment in older patients with breast cancer. J Natl Compr Canc Netw. 2009; 7:226-36.

23. Caillet P, Canoui-Poitrine F, Vouriot J, Berle M, Reinald N, Krypciak S, et al. Comprehensive geriatric assessment in the decision-making process in elderly patients with cancer: ELCAPA study. J Clin Oncol. 2011;29:3636-42. 